\title{
Impact of familial adenomatous polyposis on young adults: Attitudes toward genetic testing, support, and information needs
}

Lesley Andrews, MBBS, MMRHHG ${ }^{1}$, Shab Mireskandari, BScHons, DPsyc ${ }^{2,3}$, Jaime Jessen, BSc, MGenCouns ${ }^{2,4}$, Belinda Thewes, MClinPsych, $P h D^{2}$, Michael Solomon, MSc, FRACS ${ }^{5}$, Finlay Macrae, FRCP, $M D^{6}$, and Bettina Meiser, $B A\left(\right.$ Hons), $P h D^{7,2}$

\begin{abstract}
Purpose: The study assessed views concerning genetic testing and information and support needs among young adults aged 18 to 35 years with a diagnosis of or at risk of developing familial adenomatous polyposis. Methods: A total of 88 participants were recruited through Hereditary Bowel Cancer Registries and assessed using self-administered questionnaires. Results: The average age of participants was 28 years, and the average age at the time of their last genetic consultation was 23 years. Although $75 \%$ would consider prenatal genetic testing, only $21 \%$ would consider termination of an affected pregnancy. Sixty-one percent selected "at birth" or "early childhood" as the preferred age for genetic testing of offspring. Participants' highest areas of unmet support needs were with regard to anxiety about their children having familial adenomatous polyposis (39\%) and fear of developing cancer (28\%). Conclusion: The parental desire to test children before it is clinically indicated may be a source of distress and create conflict with genetic services. These findings demonstrate that familial adenomatous polyposis may significantly impact young adults, with many having unmet support needs. The length of time since the last genetic consultation and the young age at which these consultations took place suggest that clinics should consider a means of regular follow-up to address these unmet needs. Genet Med 2006:8(11):697-703.
\end{abstract}

Familial adenomatous polyposis (FAP) is a unique hereditary cancer syndrome with the typical onset of polyposis occurring approximately at the age of puberty. Predictive testing for a known family mutation, or surveillance sigmoidoscopy or colonoscopy, commences at approximately the same age for those at risk, and preventive colectomy is commonly required before a person is approximately 25 years of age.

\section{ISSUES IN RELATION TO GENETIC TESTING FOR FAMILIAL ADENOMATOUS POLYPOSIS}

In general, predictive genetic testing is offered only to autonomous adults after counseling and consent. It is accepted, From the ${ }^{1}$ Hereditary Cancer Clinic, Prince of Wales Hospital, Sydney, Australia; ${ }^{2}$ Psychos-
ocial Research Group, Prince of Wales Hospital, Sydney, Australia; ${ }^{3}$ Prince of Wales Clinical
School, University of New South Wales, Sydney, Australia; ${ }^{4}$ School of Biomedical Sciences,
University of Newcastle, Newcastle, Australia; ${ }^{5}$ Discipline of Surgery, University of Sydney,
Sydney, Australia; ${ }^{6}$ Department of Colorectal Medicine and Genetics, Royal Melbourne Hos-
pital, Melbourne, Australia; and ${ }^{7}$ School of Psychiatry, University of New South Wales,
Sydney, Australia.
Lesley Andrews, MBBS, Dip Obs., M.Med, Hereditary Cancer Clinic, Prince of Wales Hos-
pital, Randwick, NSW 2031, Australia.
Submitted for publication May 21, 2006.
Accepted for publication August 10, 2006.
Conflict of interest notification: The authors declare no conflict of interest with information
presented within this article.

DOI: 10.1097/01.gim.0000245574.75877.b9 however, that testing should be offered earlier if there is a demonstrated medical benefit to the knowledge. This is the case in FAP, in which predictive testing for a known family mutation is appropriate at approximately the age of puberty to enable timely colonic surveillance and management of polyposis. However, there is concern about predictive genetic testing of minors for several reasons. First, it removes the individual's right to make an autonomous decision to be tested as an intellectually competent adult. Second, it denies them the right to confidentiality of results from parents and other family members. Third, identification of a minor as carrying a mutation has the potential for adverse emotional and psychologic impact on the child. ${ }^{1}$ Finally, there is the potential for discrimination with regard to insurance and employment as adults. ${ }^{2}$

In the case of FAP, these potentially adverse outcomes are balanced against the medical benefits of early intervention. Although regular colon surveillance can be undertaken for all adolescents with a 1 in 2 risk of inheriting the gene mutation, these procedures impose a risk of medical morbidity and may be associated with psychologic distress, particularly in this population given their vulnerable age. Predictive testing enables half of those at risk to be freed from the necessity of this intense screening process. In addition, predictive testing alleviates, particularly for noncarriers and their parents, the psychologic distress of not knowing their genetic status, although this in itself is not justification for the testing of minors. Cur- 
rent practice, therefore, is to offer predictive testing for FAP to at-risk individuals between the ages of 11 and 15 years.

Several studies have examined the short- to medium-term impact of undergoing genetic testing for FAP in children and adults. ${ }^{1,3}$ Although it seems that the majority of children and adults are not adversely affected by genetic testing for FAP, there is evidence that some individuals experience clinically significant levels of psychologic morbidity after testing.

\section{PERCEIVED DISCRIMINATION EXPERIENCED BY PEOPLE WITH GENETICALLY INHERITED CANCER SYNDROMES}

In recent years there have been increasing reports of discrimination against individuals on the basis of the results of genetic testing in Australia ${ }^{4}$ and overseas. ${ }^{5,6}$ Documented cases of discrimination have occurred in the area of employment; access to health services (e.g., in vitro fertilization); and access to travel, health, income protection, life insurance, and credit. In one of the most comprehensive surveys of its kind in Australia, Barlow-Stewart and Keays ${ }^{2}$ identified 48 cases of alleged discrimination on the basis of a genetic testing result. Two of these cases involved asymptomatic FAP mutation carriers aged 20 to 29 years. There is some evidence that discrimination on the basis of genetic testing results can deter at-risk individuals from seeking genetic testing. ${ }^{2}$ For a condition such as FAP, for which interventions improve outcome and/or mortality, this finding is of significant concern. A better understanding of the nature and prevalence of genetic discrimination is required to guide the development of such policies.

This study explores the attitudes of young adults affected by or at risk of developing FAP regarding the current protocols of genetic testing and attitudes toward reproductive technologies and genetic testing of unborn offspring, and assesses the information and support needs among this group. The prevalence and nature of discrimination as a result of FAP will also be investigated.

\section{MATERIALS AND METHODS}

This study was undertaken as part of a larger study in which quality of life and psychologic adjustment were also assessed, the results of which will be reported elsewhere. Eligible participants were aged between 18 and 35 years with a clinical or a genetic diagnosis of (or with a 1 in 2 risk of developing) FAP based on family history. Participants were identified through four Australian registries: the New South Wales Hereditary Bowel Cancer Registry, Queensland Familial Bowel Cancer Registry, South Australia Familial Cancer Service, and Victorian Bowel Cancer Register. Those who had previously been diagnosed with cancer were excluded from participation.

All potentially eligible individuals were initially contacted with an invitation letter sent from their associated registries inviting them to participate in the study. Individuals were asked to return an enclosed preference card to indicate their interest in participation. Individuals who agreed to participate were then contacted by the research team, and study packages including a questionnaire, consent form and a reply-paid en- velope were mailed to these participants. Reminder calls were made and replacement questionnaires were mailed as necessary.

\section{Measures}

A questionnaire was purposively designed for this study from the review of relevant literature and based on the findings of a qualitative study we previously undertook involving indepth interviews with young adults with, or at risk of developing, FAP, the results of which will be reported elsewhere. The following measures were administered.

\section{Demographic characteristics}

Information regarding age, sex, marital status, and education level were collected. Individuals were asked whether they had children presently and if they planned to have children (or more children) in the future.

\section{Medical and family history}

Details of FAP diagnosis and FAP-related surgery, genetic counseling, genetic testing, and genetic testing results were collected.

\section{Attitudes toward genetic testing}

Five items (with "yes" vs. "no" response options) assessed views regarding preimplantation genetic diagnosis, prenatal testing, termination of an affected fetus, and preferred ages for children to undergo genetic testing and to be introduced to the concept of FAP response options: "birth," "early childhood," "10 to 14 years," "14 to 18 years," "18 years and over," and "never." See Appendix for item wording.

\section{Information sources}

One item (see Appendix) assessed satisfaction with information received so far regarding FAP (response options: "not at all satisfied," "somewhat satisfied," "very much satisfied"). A second item asked participants to select from nine sources of information they had previously used in relation to FAP (participants could select more than one option), including other family members, consultation with a genetic counselor and/or clinical geneticist, consultation with a general practitioner, consultation with a surgeon/specialist, information booklet, the Internet, medical journals, newsletters, and support groups.

\section{Support needs}

Needs for support were elicited using one item ("What has been your level of help with....") in relation to 11 issues shown in Table 2, using the following response options: "Not Applicable—-this was not a problem for me." "Satisfied-I did need help with this, but my need for help has been satisfied." "Low Need-this caused me concern or discomfort. I had little need for additional help." "Moderate Need-this item caused me concern or discomfort. I had some need for additional help." "High Need-this item caused me concern or discomfort. I had a strong need for additional help." Cronbach's alpha for the scale was found to be excellent $(\alpha=0.90)$. Two addi- 
Table 1

Summary characteristics of participants $(\mathrm{N}=88)$

\begin{tabular}{|c|c|}
\hline Variable & $\mathrm{N}(\%)$ \\
\hline \multicolumn{2}{|l|}{ Age (y) } \\
\hline $18-23$ & $22(25.3)$ \\
\hline $24-29$ & $29(33.3)$ \\
\hline $30-35$ & $36(41.4)$ \\
\hline \multicolumn{2}{|l|}{ Sex: } \\
\hline Male & $25(28.4)$ \\
\hline Female & $63(71.6)$ \\
\hline \multicolumn{2}{|l|}{ Marital status: } \\
\hline Single/separated/divorced & $30(34.5)$ \\
\hline Married/de facto & $57(65.5)$ \\
\hline \multicolumn{2}{|l|}{ Children: } \\
\hline No & $47(54.0)$ \\
\hline Currently pregnant & $3(3.4)$ \\
\hline Yes & $37(42.5)$ \\
\hline \multicolumn{2}{|l|}{ FAP status: } \\
\hline \multicolumn{2}{|l|}{ Clinically unaffected $(\mathrm{N}=17)$} \\
\hline Mutation carrier & $2(11.8)$ \\
\hline At 1 in 2 risk & $15(88.2)$ \\
\hline \multicolumn{2}{|l|}{ Clinically affected $(\mathrm{N}=71)$} \\
\hline Had surgery & $57(81.4)$ \\
\hline No surgery yet & $14(19.7)$ \\
\hline \multicolumn{2}{|l|}{ Surgery status $(N=57)$ : } \\
\hline Total colectomy and IRA & $33(57.9)$ \\
\hline Restorative proctocolectomy & $16(28.1)$ \\
\hline Proctocolectomy and ileostomy & $1(1.8)$ \\
\hline $\begin{array}{l}\text { Colectomy and } \\
\text { proctocolectomy }\end{array}$ & $5(8.8)$ \\
\hline Do not know what type surgery & $2(3.5)$ \\
\hline
\end{tabular}

FAP, familial adenomatous polyposis; IRA, ileorectal anastomosis.

tional open-ended questions asked participants if they had experienced any other needs for support about FAP and whether they had suggestions for improving services for individuals at risk of FAP or diagnosed with FAP.

\section{Preferred methods of information/support provision}

One item (see Appendix) asked participants to rank their preferences from 1 (most preferred) to 8 (least preferred) for eight methods of service delivery shown in Table 3. Each information/support provision tool was followed with a brief description.

\section{Discrimination}

One open-ended question asked participants about possible experiences of discrimination as a result of being at risk of FAP or being diagnosed with FAP.
Table 2

Proportion of participants reporting moderate to high levels of unmet support needs $(\mathrm{N}=88)$

\begin{tabular}{|c|c|c|}
\hline Item & $\begin{array}{l}\text { Number of participants } \\
\text { reporting moderate to } \\
\text { high levels of unmet } \\
\text { need }\end{array}$ & $\begin{array}{l}\text { Percentage of } \\
\text { sample } \\
\text { reporting moderate } \\
\text { to high levels of } \\
\text { unmet need }\end{array}$ \\
\hline $\begin{array}{l}\text { Dealing with worry/anxiety } \\
\text { about your children } \\
\text { developing FAP }\end{array}$ & 34 & 38.6 \\
\hline $\begin{array}{l}\text { Dealing with fears about } \\
\text { developing cancer }\end{array}$ & 25 & 28.4 \\
\hline $\begin{array}{l}\text { Dealing with uncertainty } \\
\text { about the impact of FAP }\end{array}$ & 21 & 23.9 \\
\hline $\begin{array}{l}\text { Dealing with the impact of } \\
\text { FAP on the family }\end{array}$ & 20 & 22.7 \\
\hline $\begin{array}{l}\text { Obtaining information } \\
\text { about FAP }\end{array}$ & 18 & 20.5 \\
\hline $\begin{array}{l}\text { Understanding } \\
\text { information given }\end{array}$ & 16 & 18.2 \\
\hline $\begin{array}{l}\text { Reassurance that the way } \\
\text { you feel is normal }\end{array}$ & 15 & 17.0 \\
\hline $\begin{array}{l}\text { Dealing with the loss of } \\
\text { family members who } \\
\text { died of cancer }\end{array}$ & 14 & 15.9 \\
\hline $\begin{array}{l}\text { Talking with other people } \\
\text { who are at risk of or } \\
\text { diagnosed with FAP }\end{array}$ & 13 & 14.8 \\
\hline $\begin{array}{l}\text { Dealing with sadness } \\
\text { about FAP }\end{array}$ & 11 & 12.5 \\
\hline
\end{tabular}

FAP, familial adenomatous polyposis.

\section{Statistical analysis}

Data were analyzed using the Statistical Package for the Social Sciences 14 (SPSS Inc., Chicago, IL). Descriptive statistics were used to describe the sample in terms of sociodemographic and clinical characteristics, and their attitudes toward genetic testing and information and support needs. Differences in attitudes and information-related outcomes between participants who previously had genetic counseling and those who had not were explored using chi-square tests. To explore differences in unmet support needs depending on genetic counseling status, a mean unmet needs score was calculated using the support needs scale, and a Mann Whitney $U$ test was performed to test for differences between groups.

\section{RESULTS}

\section{Sociodemographic and medical characteristics}

Of 265 registrants sent invitations to participate, 108 were lost to contact and 11 were found to be ineligible. Of the remaining 146 registrants whose current address details were available, 116 consented to be contacted; of those, 88 returned the completed questionnaire, resulting in an overall participation rate of $60.3 \%$ among those who were successfully contacted. 
Table 3

Preferences for familial adenomatous polyposis-related information $(\mathrm{N}=88)$

\begin{tabular}{lccc}
\hline \multirow{2}{*}{$\begin{array}{c}\text { Communication } \\
\text { strategy/information tool }\end{array}$} & Ranking No. & \multicolumn{2}{c}{$\begin{array}{c}\text { Preference } \\
\text { rating }\end{array}$} \\
\cline { 3 - 4 } Consultation with medical expert & 1 & 2.7 & 1.9 \\
Consultation with genetic expert & 2 & 3.2 & 1.8 \\
$\begin{array}{l}\text { Talks/information sessions by } \\
\text { experts }\end{array}$ & 3 & 4.4 & 2.0 \\
Information/pamphlet booklet & 4 & & \\
Information video/DVD & 5 & 4.8 & 2.1 \\
Internet & 6 & 4.9 & 2.1 \\
Support group & 7 & 5.3 & 2.1 \\
CD-ROM & 8 & 5.8 & 2.0 \\
\hline SD, & & &
\end{tabular}

SD, standard deviation.

Table 1 provides a summary of the sociodemographic and medical characteristics of participants. Twenty-five participants were male $(28.4 \%)$, and 63 participants were female (71.6\%). The average age of participants was 27.6 years (standard deviation $[S D]=4.9$, range $=18-35$ years). Seventy-one participants (80.7\%, 22 males and 49 females) were clinically affected by FAP, and 17 participants (19.3\%, 3 males and 14 females) were clinically unaffected by FAP. Among clinically affected individuals, 42 reported having an identified mutation, 5 reported having received inconclusive results, 15 reported that they had not undergone genetic testing, and 6 reported that they did not know whether or not they had undergone genetic testing. Among clinically unaffected individuals, 2 reported being known carriers of the family mutation; 2 reported having received inconclusive results; and 13 reported not having undergone genetic testing; but these were known to have a 1 in 2 risk of carrying a mutation. Of the 71 participants clinically affected by FAP, 14 (15.9\%) had not undergone surgery. Fifty-four participants $(63.5 \%)$ had consulted with a genetic counselor or clinical geneticist regarding FAP, 27 (31.8\%) had not consulted a genetic counselor or clinical geneticist, and 4 (4.5\%) did not know if they had consulted either. The average age at the last genetic consultation was 22.6 years ( $\mathrm{SD}=6.4$, range $8-34$ years). The average age of participants when FAP was first identified in the family was 12.6 years $(S D=10.3$, range $=$ before birth to 32 years $)$, and the average age at the time of genetic testing was 21.4 years $(\mathrm{SD}=$ 6.3 , range $11-34$ years).

\section{Attitudes toward genetic testing}

In regard to attitudes toward genetic testing, $75 \%$ of participants reported that they would consider prenatal testing for FAP. In addition, $61.4 \%$ would consider preimplantation genetic diagnosis. In contrast with this, 20.9 would consider termination of a fetus found to carry a FAP-related mutation. In regard to genetic testing for children, $42 \%$ selected testing at birth, 19.3\% selected testing during early childhood, $18.2 \%$ selected testing at 10 to 14 years, $18.2 \%$ selected testing at 14 to 18 years, and $1.1 \%$ preferred 18 years or older as the most appropriate time for genetic testing. In terms of age at which to introduce children to the concept of FAP, $34.1 \%$ preferred early childhood, $42.4 \%$ preferred 10 to 14 years, $20.0 \%$ preferred 14 to 18 years, and $3.5 \%$ preferred 18 years or more. No statistically significant differences in attitudes toward genetic testing were observed depending on whether participants had previously undergone genetic counseling.

In response to the open-ended question regarding suggestions for improving services, one single mother said she would like to know her child's genetic status before having further children. Another participant commented that "[It] should be the right of the parent to decide what age [children] can be tested, not the Hereditary Cancer Register to dictate the rights of someone else's life."

\section{Support needs}

Table 2 shows the data on perceived need for support in regard to specific issues relating to FAP. The three most frequently endorsed areas in which participants reported having moderate/high levels of need for support were as follows: dealing with (1) anxiety related to their children developing FAP (38.6\%), (2) fears of developing cancer $(28.4 \%)$, and (3) uncertainty about the impact of FAP (23.9\%). Overall, $62 \%$ of participants expressed a moderate or high level of need for support in one or more areas. In response to open-ended questions regarding service improvements, several participants requested support groups to be set up locally or on the Internet. No statistically significant differences in levels of support needs were observed depending on whether participants had previously undergone genetic counseling.

\section{Information needs}

It was found that the majority of participants (94.3\%) were either "somewhat satisfied" or "very much satisfied" with the level of information they had received so far with regard to FAP. No statistically significant differences in satisfaction were found depending on whether participants had previously received genetic counseling. Seventy-seven percent (77.3\%) had used other family members to access information about FAP; $73.9 \%$ had used information booklets or pamphlets; $70.5 \%$ asked a surgeon or specialist; $55.7 \%$ asked a genetic counselor or geneticist; $38.6 \%$ asked a general practitioner; $34.1 \%$ used the Internet; $25.0 \%$ read newsletters; $4.5 \%$ read medical journals; and $4.5 \%$ had been involved in support groups.

Table 3 shows the data on the preferred sources of FAPrelated information provision types. The most preferred source of information in regard to FAP was consultation with a medical expert, such as a surgeon or gastroenterologist, with consultation with a genetic expert selected as the second most preferred option. This was followed by talks/information sessions held by experts on FAP and then the provision of information pamphlet/booklets summarizing the major issues related to FAP. 
In response to the open-ended question in regard to suggestions for improving services, participants proposed information sessions in rural areas and opportunities for contacting other young people affected with FAP either in person or through a chat room on the Internet.

\section{Discrimination}

Fourteen participants (16.3\%) reported that they had experienced discrimination as a result of FAP. The majority of reports concerned employment (seven reports), including the need for frequent toilet breaks, time off from work for surveillance, and physical limitations adversely affecting job opportunities and pay increases; these factors were seen as having the potential to give rise to negative attitudes from colleagues and managers. Problems were also reported obtaining life insurance (two reports), sickness benefits (one report), and private health insurance (one report). Three participants reported social discrimination caused by a lack of understanding by others and stares when going to the beach because of their surgery scars. No participant reported taking any form of redress for perceived discrimination.

\section{DISCUSSION}

The typical onset of polyposis and the subsequent introduction of interventions in adolescence make FAP a unique cancer predisposition syndrome warranting close examination of the management of these individuals at all ages. Unfortunately, the rarity of the syndrome and the difficulties associated with the research of minors have resulted in a shortage of evidence on which to base recommendations for standard practice. Although genetic testing for known family mutations is accepted practice in early adolescence, there is little evidence on which to base guidelines for ongoing follow-up by genetic services for carriers or for those who remain at risk if no family mutation is known. In addition, there are few data to guide clinicians on the need for referral of new mutation carriers for genetic services.

In this study, we found that three quarters $(75.0 \%)$ of participants would consider prenatal testing for FAP, but that only one fifth $(20.9 \%)$ would consider termination of an affected pregnancy. These findings are similar to those of Whitelaw et al., 7 who found that among a group of patients with FAP with an average age of 38 years, $65 \%$ would request prenatal testing, and of these only $24 \%$ stated that they would proceed to termination of pregnancy if the fetus was affected. It is of interest that a decade later, there has not been a change in desire for termination among potential parents of FAP-affected children. Taken together, however, both studies support the conclusion that despite accepted guidelines, potential parents have a strong desire to know the genetic status of their children, without any immediate health benefit to the child. Answers to open-ended questions indicated that some parents strongly resent that current guidelines do not respect their parental rights. Thus, clinic refusal to offer genetic testing of infants may alienate some couples from genetic services altogether.
Despite the low interest in procurement of pregnancy termination, our findings indicate an interest in using other means of trying to avoid having an affected child. Almost two thirds of participants (61.4\%) in the current study would consider preimplantation genetic diagnosis. This correlates with our data that the support these individuals most require is with the worry and anxiety about their children developing FAP. Our findings that the greatest need for support was in regard to the impact of FAP on their children and the high level of desire for knowledge of the genetic status of their offspring indicate that these issues require more counseling. Because the average age at last genetic consultation for this sample was 23 years, it is possible that past genetic counseling has not sufficiently addressed the wide range of issues pertaining to childbearing. This reinforces the need for genetic follow-up and periodic review in addition to regular medical follow-up among this population.

Two thirds of our cohort reported preferring children to undergo genetic testing at birth or early childhood, much before the accepted current practice. In the study by Whitelaw et al., ${ }^{7}$ the percentage of participants who indicated that their preferred age of testing children was at birth or in early infancy was even higher (93\%). The lack of medical interventions at this age indicates a desire to know for knowledge's sake, perhaps seeking the reassurance that the offspring is not affected. Because more than half of this cohort had undergone genetic testing themselves, it is reassuring that only one individual preferred genetic testing to be postponed until after the age of 18 years, indicating that those who have undergone testing themselves do not view genetic testing of minors as disadvantageous or harmful. Despite the intended desire to know the offspring's genetic status, almost half of participants thought the preferred age for discussing FAP with their children was between 10 and 14 years, which is the usual age for testing or screening. The age gap between the preferred age for genetic testing as being infancy or early childhood and the preferred age for discussing FAP with children being 10 to 14 years reinforce the view that many parents have a strong desire to know genetic status but do not want their children to be burdened with this knowledge before it is actually necessary. It is important to also note that one third (34.1\%) of the sample reported that they would discuss FAP with their children before the age of 10 years. Given that this is before the age of genetic counseling and testing, these parents may need to be given information and counseling themselves to be ready for the potentially distressing task of talking with their young children about FAP on their own. Of great concern is that one fourth of participants (23.5\%) reported they would not discuss FAP with their children until after the age of 14 years, when there is already considerable risk of polyps. Further research is required to investigate possible reasons for this view and to find solutions on how to address this.

The discrepancy between the desired age of testing children and the accepted current practice is likely to cause distress for parents and geneticists alike, particularly as consumer rights increase. Without a sound relationship between genetic services and parents, families may feel unsupported at times of 
significant distress, particularly when testing of offspring does occur. As parents hope for a negative test result for their own children, they may be less likely to be aware of the potential negative impact of a positive test result. ${ }^{8}$ Petersen and Boyd ${ }^{9}$ found that many parents did not anticipate the stress that genetic testing adds to a child's life and their own lives, and that they are not necessarily an accurate gauge by which to judge a child's understanding of FAP. This study found parents commonly overestimated or underestimated their child's knowledge of FAP. ${ }^{9}$ Care must therefore be taken to address the needs of parents seeking early genetic testing in a way that minimizes harm to all family members and maintains a sound relationship with genetics services.

Participants in this study expressed a high level of need for FAP-related support, with almost two thirds expressing a moderate or high need for support in some area, which indicates a wide spread of areas with unmet need. Almost two thirds of the participants (63.5\%) previously had a consultation with a geneticist or genetic counselor, with the last consultation being on average 5 years ago. For half of these young adults, their last genetic consultation was conducted before the age of 23 years. The information provided at these consultations would not typically cover many of the issues that need to be confronted in the late 20 s and 30 s, such as childbearing. This indicates a need for genetic services or advice through medical specialists to be offered on an ongoing basis and not to be confined to the issues surrounding genetic testing and immediate medical management. Although the majority of participants identified a medical specialist as the preferred source of support, this is perhaps not surprising given that one third (31.8\%) had never seen a geneticist/genetic counselor and that $70.5 \%$ had consulted with a medical specialist. Because $80.7 \%$ of the participants are clinically affected, it is likely that these people are having regular medical surveillance, but despite this, their support needs are not being met, indicating that additional sources may be needed. Seventy-seven percent of this cohort had used other family members as a source of information, and of particular concern are those young adults who are apparently carriers of a de novo mutation, because these individuals will not have the benefit of previous family experience of FAP. Even if genetic testing is not required for the patient's immediate management, referral to a familial cancer clinic can provide ongoing education and support, helping these individuals address the concerns identified in this study, which relate not only to themselves but also to their future offspring as well.

Other studies support the notion that the lengthy time since the last genetic consultation may be a factor in the high level of unmet needs. It has been found that that those who have had genetic counseling recall only approximately two thirds of the medical and risk information presented. ${ }^{10}$ In addition to this, many of those who undergo genetic testing or have a clinical diagnosis of FAP do so in adolescence. Well-recognized theories of cognitive development demonstrate that children are unable to understand the full implications of genetic testing until they reach the stage of formal operational thought, which typically begins around the age of 11 years or possibly several years later. ${ }^{8}$ Because genetic testing is offered typically from the ages of 10 to 13 years, those at risk of FAP may receive genetic counseling at a time when comprehension of health-related procedures and risk is not fully developed, and their ability to process the complex emotional issues is limited.

Participants in the current study were asked to rank their preference for different types of communication strategies for information about FAP. The top two preferred sources of information were consultations with both medical experts and genetic experts. Talks and information sessions were also highly ranked. Because of the young age of these participants and current technology available, it was surprising that CDROMs, the Internet, and video/DVDs were not preferred by these individuals. A CD-ROM was reported to be least preferred within this group. These findings indicate that it is the interactive process of the consultation that is important, not just the provision of information.

Only two participants reported discrimination with regard to life insurance. This figure is low considering the sample included 57 individuals affected by FAP who would be denied life insurance under existing Australian law. This could be because of the young age of the cohort, resulting in a low interest in life insurance, or because individuals with FAP have not bothered seeking insurance because they know it will be denied. Several reports of work-related discrimination cite a poor understanding of FAP by employers. A short brochure explaining FAP for managers may be useful in helping individuals with FAP obtain optimal employment.

\section{LIMITATIONS}

This study had a cross-sectional design, and it would be useful to conduct this research prospectively with participants in early adolescence. Although FAP is not a sex-specific disease, the majority $(72.4 \%)$ of our participants were female, and therefore data may not accurately represent the psychosocial impact of FAP on males within this age group. Furthermore, only individuals who were listed in a FAP registry were included in this study. This is a voluntary process, and those individuals who chose not to join the registry may in fact have much different attitudes and levels of unmet needs. There was a high proportion of registrants who were lost to contact (108/ 265), and these registrants may have different responses than those who have stayed in contact with the registers.

\section{CONCLUSIONS}

Although the primary management of FAP is surgical, this study highlights the importance of the role of genetic services. The marked discrepancy between parental desire for knowledge of the genetic status of their offspring from birth and the guidelines of genetic authorities indicates the importance of individual counseling of all couples to reach an outcome that is acceptable to both. Because of the high interest in preimplantation genetic diagnosis, knowledge of the availability, limitations, and cost of such advanced techniques should be available 
to all prospective parents to enable them to make informed choices. Current genetic services could be improved by ensuring that clients are offered periodic review, especially for those whose last contact was in adolescence. Finally, discrimination in the workplace may be alleviated by better written materials, especially designed to communicate accurate information to employers and others.

\section{APPENDIX: ITEMS ASSESSING ATTITUDES AND INFORMATION NEEDS}

Attitudes toward genetic testing:

If you knew it was possible to detect familial adenomatous polyposis (FAP) in embryos conceived through in vitro fertilization, would you consider this test to avoid having a FAPaffected child?

If you knew it was possible to detect in the early weeks of pregnancy whether your child had the gene change associated with FAP, would you be interested in having this test?

If the test showed that the unborn baby carried the gene change associated with FAP, would you be in favor of terminating the pregnancy?

In many cases it is possible to detect the gene associated with FAP by performing a blood test. At what age would you want your own children to be tested?

At what age do you think children at risk of FAP should be introduced to the concept of FAP?

Information sources:

How satisfied are you with the level of information you have so far received regarding FAP?

Preferred methods of information/support provision:

For us to improve the services provided, we would like to know how useful you think the following resources would be or have been to you. Once you have considered each option, please rank the options from the one that you most prefer $($ rank order $=1)$ to the one that you least prefer ( rank order $=8$ ).

\section{ACKNOWLEDGMENTS}

We are most grateful for the valuable contribution of all those who participated in this study. The authors thank the following individuals for their assistance with participant ascertainment and recruitment: NSW and ACT Hereditary Cancer Registers, especially Freddy Sitas, Sarah Doak, and Udani Abeypala; Queensland Familial Bowel Cancer Registry, especially Ngaire Knight and Tricia Keevers; Familial Cancer Unit, South Australian Clinical Genetics Service, especially Dr. Graeme Suthers; and Victorian Family Cancer Register, especially Marita Black, Toula McArdle, and Kate Minkoff. This study was funded by the Cure Cancer Foundation of Australia. Dr. Bettina Meiser is supported by a Career Development Award from The National Health and Medical Research Council of Australia (ID 350989).

\section{References}

1. Codori A, Zawacki K, Petersen G, Miglioretti, DL, et al. Genetic testing for hereditary colorectal cancer in children: long-term psychological effects. Am J Med Genet 2003;116A:117-128.

2. Barlow-Stewart K, Keays D. Genetic discrimination in Australia. J Law Med 2001; 8(3):250-262.

3. Codori A, Petersen GM, Boyd PA, Brandt J, et al. Genetic testing for cancer in children: short-term psychological effect. Arch Pediatr Adolesc Med 1996;150:11311138 .

4. Otlowski MF, Taylor SD, Barlow-Stewart KK. Genetic discrimination: too few data. Eur J Hum Genet 2003;11(1):1-2.

5. Geller L, Alper J, Billings P, Barash C, et al. Individual, family, and societal dimensions of genetic discrimination: a case study analysis. Sci Eng Ethics 1996;2(1):71-88.

6. Low L, King S, Wilkie T. Genetic discrimination in life insurance: empirical evidence from a cross sectional survey of genetic support groups in the United Kingdom. BMJ 1998;317(7173):1632-1635.

7. Whitelaw S, Northover JM, Hodgson SV. Attitudes to predictive DNA testing in familial adenomatous polyposis. J Med Genet 1996;33:540-543.

8. Wertz D, Fanos JH, Reilly PR. Genetic testing for children and adolescents: who decides? JAMA 1994;272(11):875-881.

9. Petersen GM, Boyd PA. Gene tests and counseling for colorectal cancer risk: lessons from familial polyposis. J Natl Cancer Inst Monogr 1995;17:67-71.

10. Michie S, French D, Allanson A, Bobrow M, et al. Information recall in genetic counselling: a pilot study of its assessment. Pat Educ Couns 1997;32:93-100. 